\title{
Eulobomyia, a new replacement name for Lobomyia Woodley \& Arnaud (Diptera: Tachinidae)
}

\author{
NORMAN E. WOODLEY ${ }^{1} \&$ PAUL H. ARNAUD, JR. ${ }^{2}$ \\ ${ }^{1}$ Systematic Entomology Laboratory, PSI, ARS, USDA, \% Smithsonian Institution NHB-168, P O Box 37012, Washington, DC 20013- \\ 7012, USA.E-mail: norman.woodley@ars.usda.gov \\ ${ }^{2}$ Department of Entomology, California Academy of Sciences, 55 Concourse Drive, San Francisco, CA 94118, USA. E-mail: par- \\ naud@calacademy.org
}

A new genus, Lobomyia Woodley \& Arnaud (Diptera: Tachinidae), from the Neotropical Region was recently described by us (Woodley \& Arnaud, 2008: 32; type species, Lobomyia neotropica Woodley \& Arnaud; published 6 June 2008 ). At the time the manuscript was being prepared, one of us (NEW) checked available nomenclatural resources to ensure the generic name had not been previously used. However, in the interim, the name Lobomyia was published as a new genus of Diptera, Chironomidae from Japan (Niitsuma, 2007: 105; type species, Lobomyia immaculata Niitsuma; published 15 June 2007). We therefore propose a new replacement name here, Eulobomyia, nom. nov. for Lobomyia Woodley \& Arnaud. The gender of the name is feminine.

We are grateful to Owen Lonsdale, Department of Entomology, Smithsonian Institution, for pointing out that Lobomyia Woodley \& Arnaud was preoccupied.

\section{References}

Woodley, N.E. \& Arnaud, P.H., Jr. (2008) Lobomyia neotropica, a new genus and species of Tachinidae (Diptera) from the Neotropical Region. Zootaxa, 1783, 31-39.

Niitsuma, H. (2007) Rheopelopia and two new genera of Tanypodinae (Diptera, Chironomidae) from Japan. Japanese Journal of Systematic Entomology, 13, 99-116. 